\title{
Substrateless Welding of Self-Assembled Silver Nanowires at Air/Water Interface
}

Hang $\mathrm{Hu}^{\dagger, \perp}$, Zhongyong Wang ${ }^{\dagger, \perp}$, Qinxian $\mathrm{Ye}^{\dagger}$, Jiaqing $\mathrm{He}^{\dagger}$, Xiao $\mathrm{Nie}^{\dagger}$, Gufeng $\mathrm{He}^{\ddagger}$,

Chengyi Song ${ }^{\dagger}$, Wen Shang ${ }^{\dagger}$, Jianbo $\mathrm{Wu}^{\dagger}$, Peng Tao ${ }^{*}$, and Tao Deng $^{*}, \dagger$

${ }^{\dagger}$ State Key Laboratory of Metal Matrix Composites, School of Materials Science and Engineering, Shanghai Jiao Tong University, Shanghai 200240, P. R. China

${ }^{\sharp}$ School of Electronic Information and Electrical Engineering, Shanghai Jiao Tong University, Shanghai 200240, P.R. China

${ }^{\perp}$ These authors contributed equally to this work.

Corresponding Authors:

*E-mail: taopeng@sjtu.edu.cn; dengtao@sjtu.edu.cn 


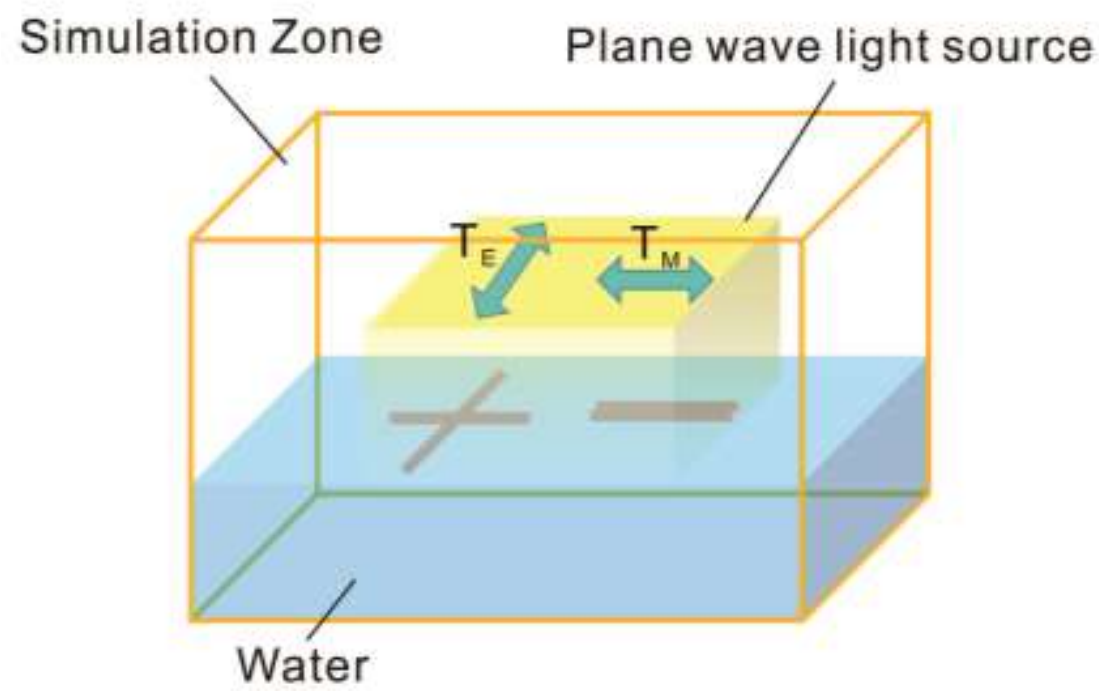

Figure S1. Schematic FDTD modeling setup for plasmonic welding at air/water interface.

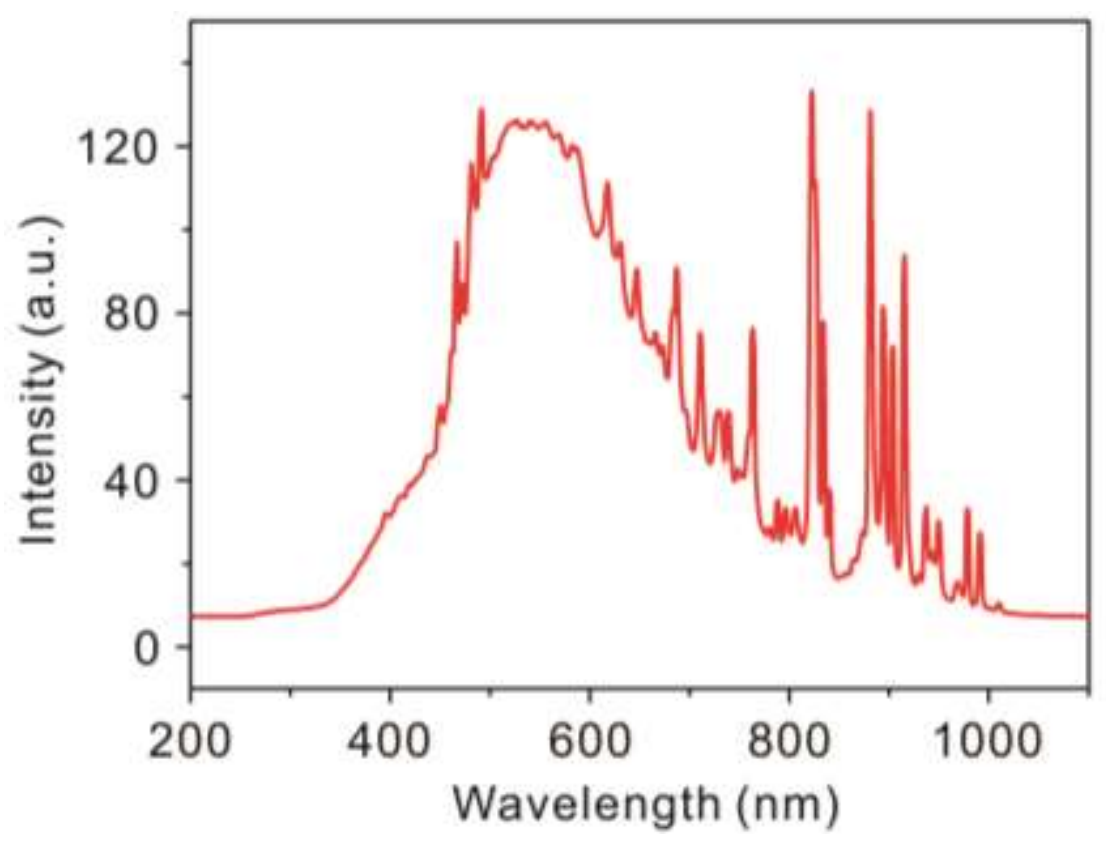

Figure S2. Illumination intensity-wavelength curve of solar simulator. 

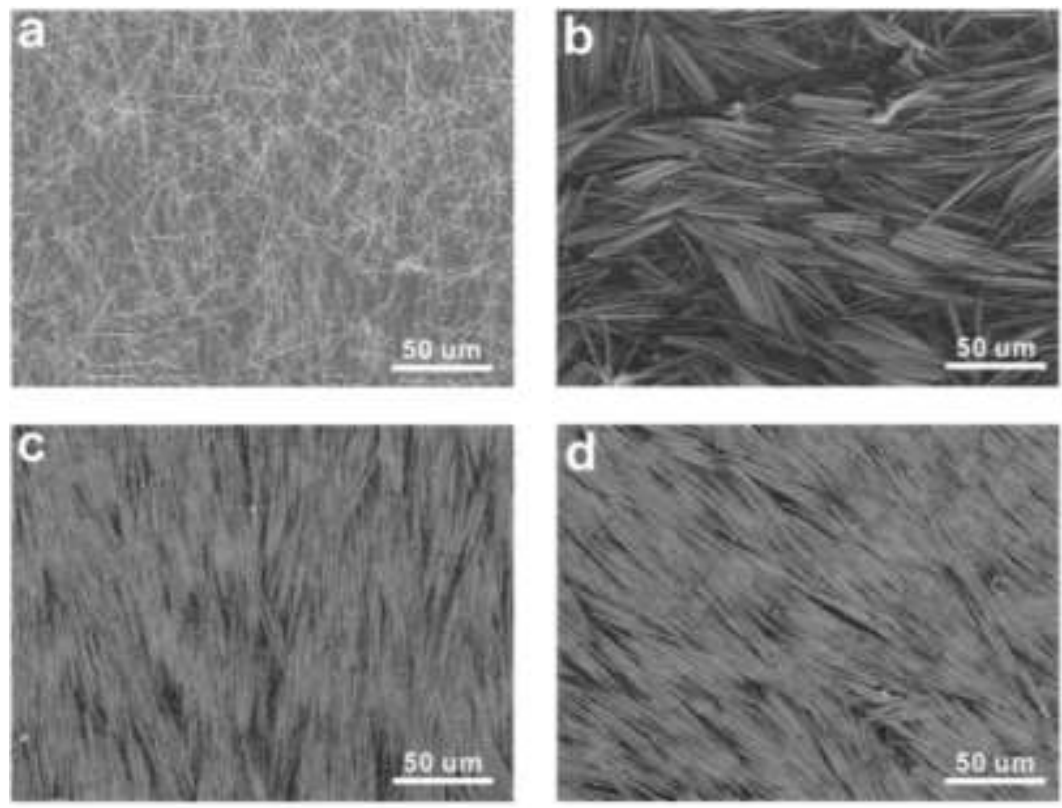

Figure S3. Microstructure of synthesized and assembled Ag NWs. (a) An SEM image of the as-synthesized Ag NW. (b, c, d) SEM images of assembled Ag NW films obtained from Ag NW dispersion with a concentration of $0.3 \mathrm{mg} / \mathrm{ml}, 0.5 \mathrm{mg} / \mathrm{ml}$ and 1 $\mathrm{mg} / \mathrm{ml}$, respectively.
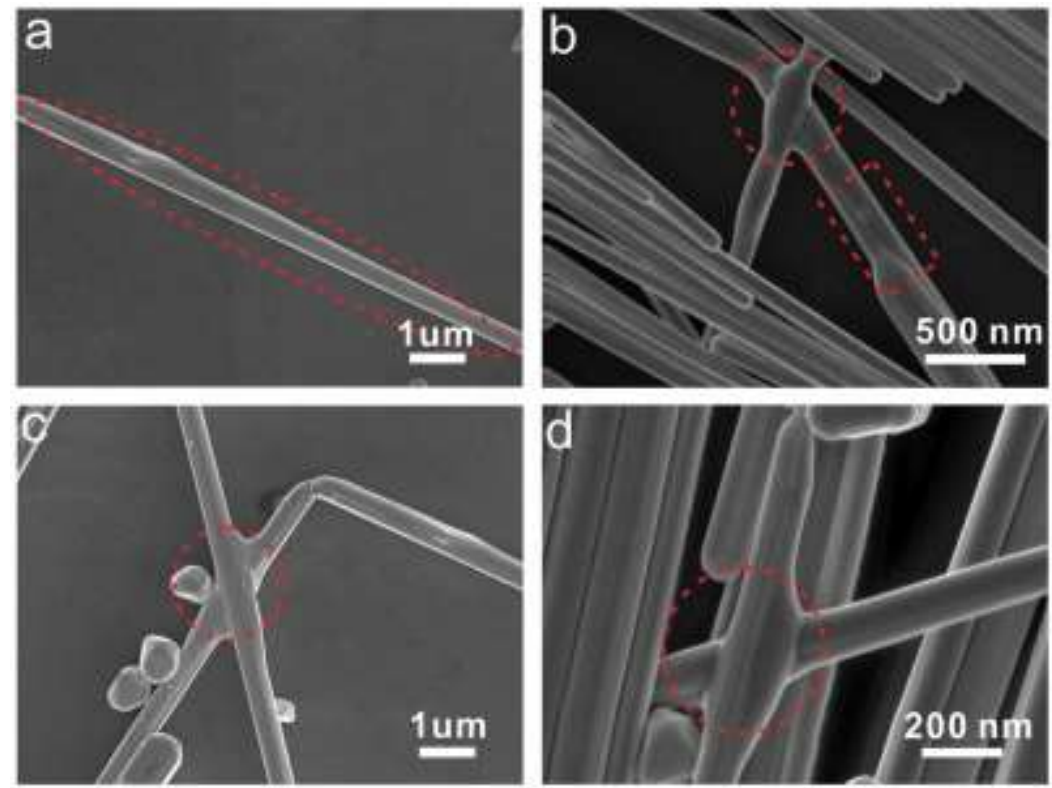

Figure S4. SEM images of welded Ag NWs with different welding structures. (a) SEM image of fusion between a single pair of Ag NWs. (b) SEM image of welding at crossed junctions and the accompanying fusion between aligned NWs. (c) SEM image of welded crossed junctions. (d) Tilted view of welded Ag NW junctions. 


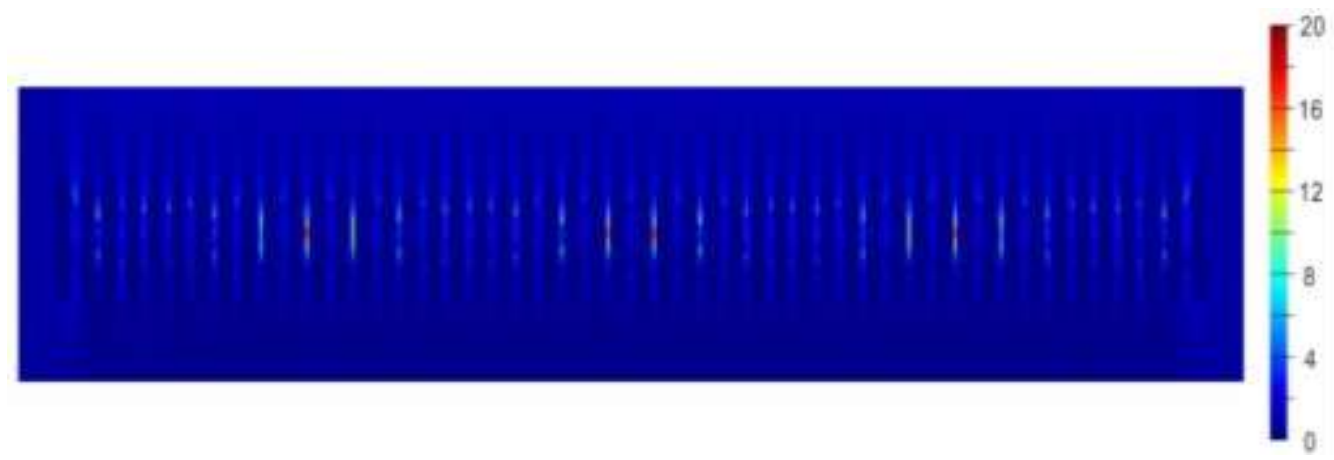

Figure S5. FDTD simulation of electric filed distribution among 50 aligned Ag NWs.
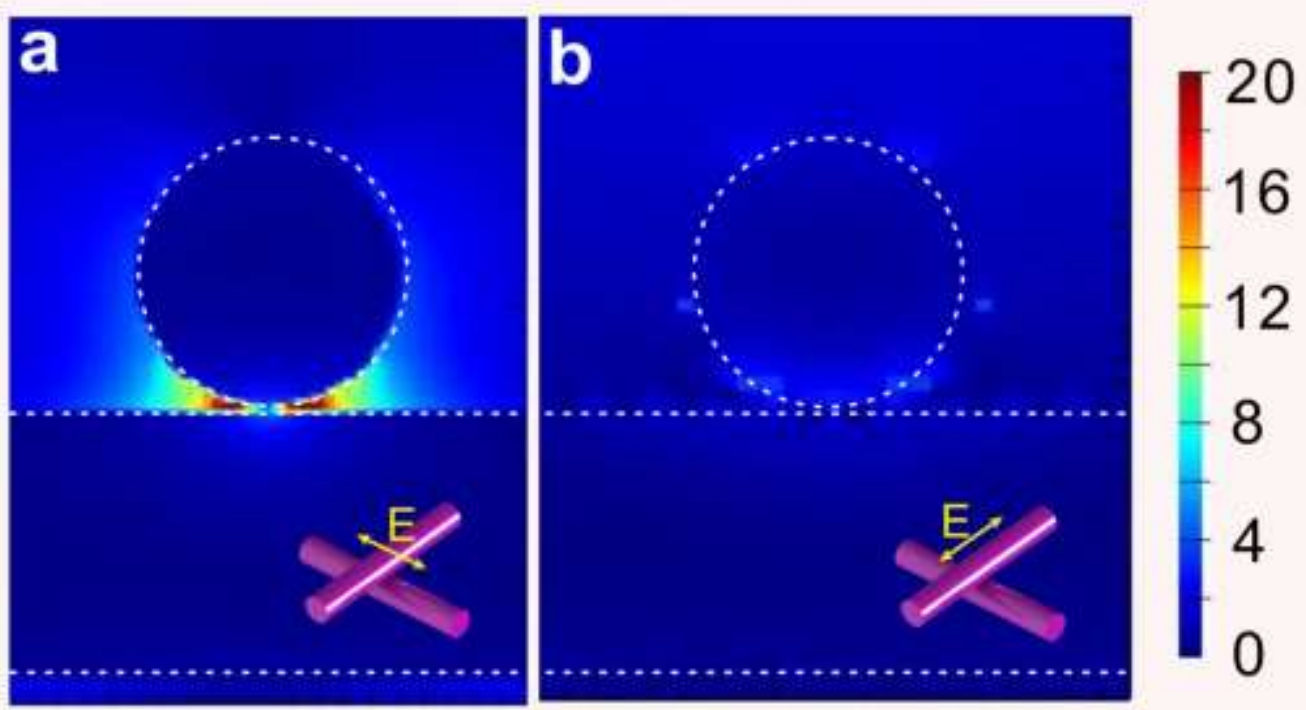

Figure S6. FDTD simulation of electric field distribution of perpendicularly crossed Ag NW junctions under excitation of light (at the wavelength of $500 \mathrm{~nm}$ ) with different polarizations: (a) perpendicular to the top NW; (b) parallel to the top NW. 


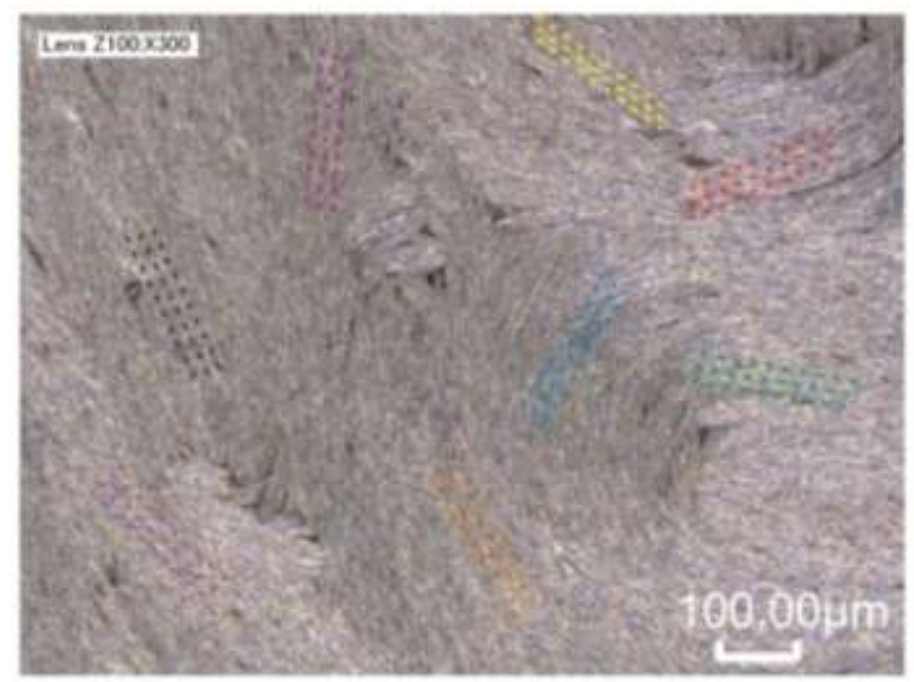

Figure S7. Optical microscope image of the self-assembled Ag NW films showing random orientations of Ag NW bundles at macro-scale. The dotted lines delineate the local orientation of assembled NWs.

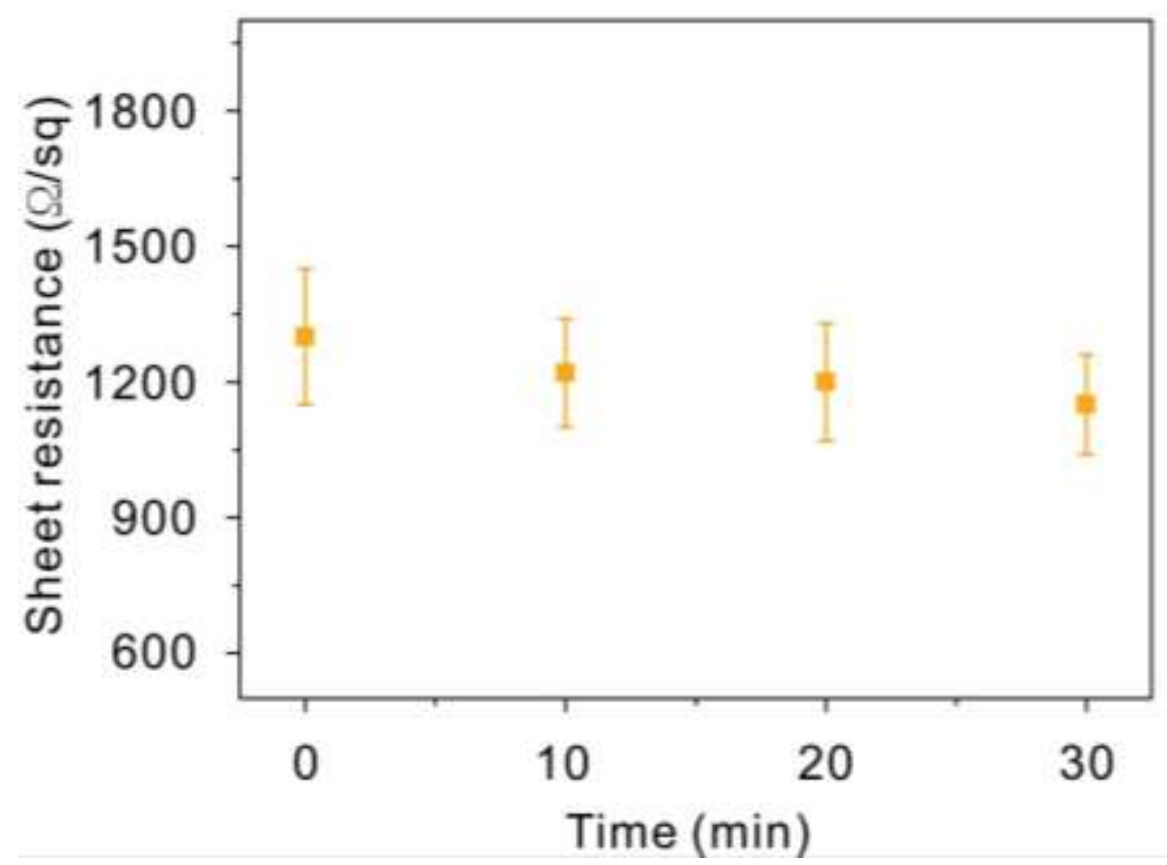

Figure S8. Sheet resistance evolution of assembled Ag NW films floating on the surface of water at $45^{\circ} \mathrm{C}$ for different durations. 

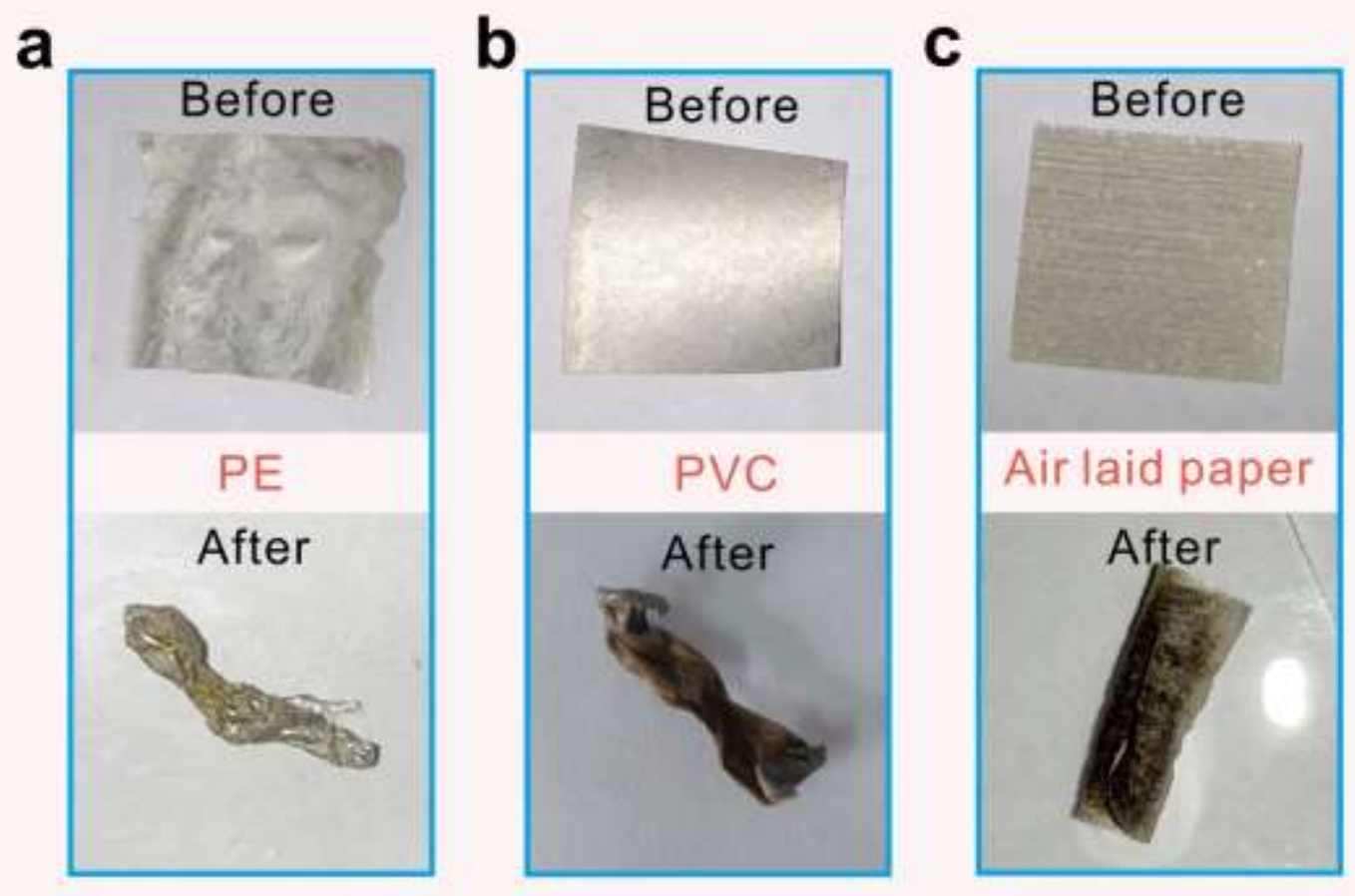

Figure S9. Plasmonic welding of Ag NWs drop-casted on heat-sensitive substrates before and after solar illumination under a power density of $15 \mathrm{~W} / \mathrm{cm}^{2}$ for $2 \mathrm{~min}$ : (a) polyethylene (PE) film; (b) polyvinylchloride (PVC) film; (c) airlaid paper.
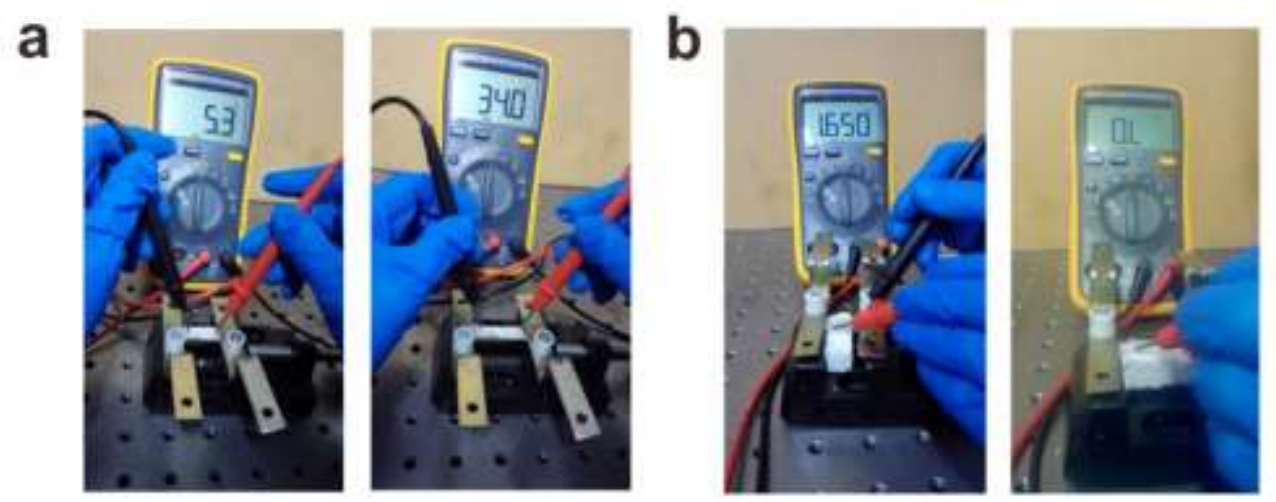

Figure S10. Comparative electric resistances of PDMS composites integrated with welded and as-assembled Ag NW films. (a) Resistance of the conductor with welded Ag NWs under applied strain of 0 (left, $5.3 \Omega$ ) and $80 \%$ (right, $34 \Omega$ ) after 50 cycles of stretching and releasing. (b) Resistance of the conductor with as-assembled $\mathrm{Ag}$ NWs under applied strain of 0 (left, $1650 \Omega$ ) and 40\% (right, breaking-down). 


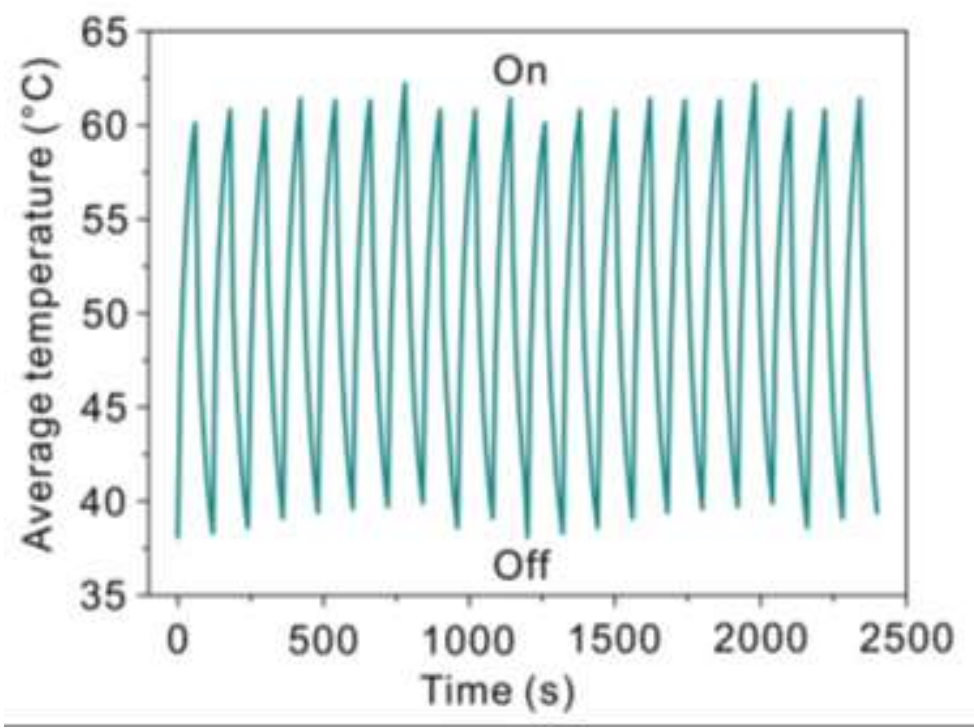

Figure S11. On/off response of PDMS-Ag NW composite heater under applied strain of $20 \%$ and DC voltage of $5 \mathrm{~V}$.
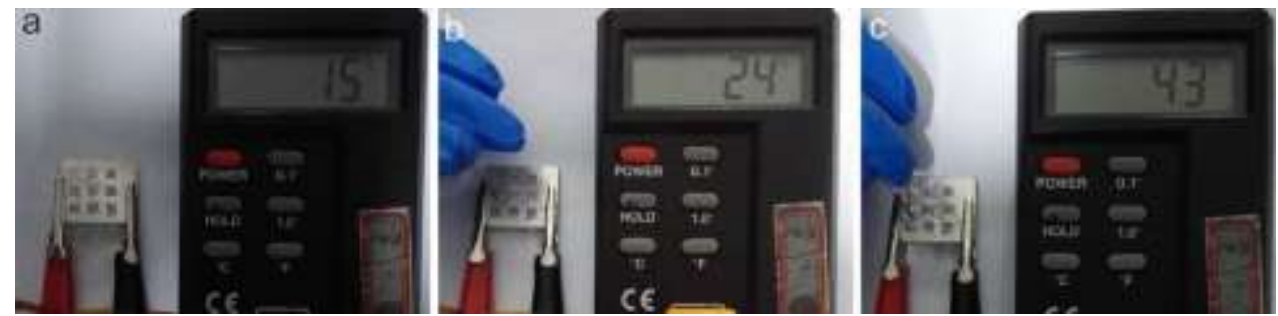

Figure S12. Temperature contrast of patterned heater under applied 5V DC voltage probed by a thermocouple. (a) Room temperature. (b) Temperature of exposed PDMS substrate. (c) Temperature of welded Ag NW film.

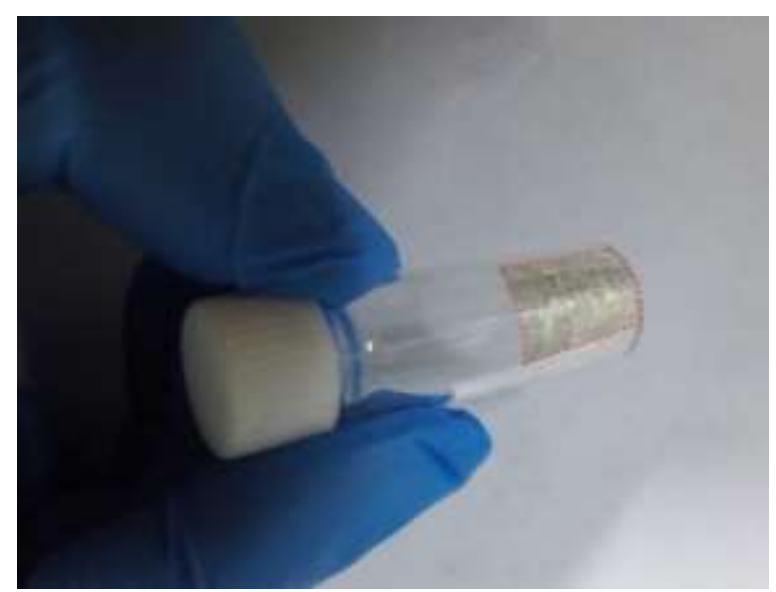

Figure S13. A photograph showing easy transfer of welded self-assembled Ag NW film onto curved glass vial surface. 